\title{
Contribuciones y efectos de los videojuegos en la atención a la diversidad
}

\section{Contributions and Effects of Video Games in Attention to} Diversity

\section{Contribuições e efeitos dos videogames na atenção à diversidade}

\author{
Silvia López-Gómez* iD orcid.org/0000-0002-5256-0793 \\ Jesús Rodríguez-Rodríguez** iD orcid.org/0000-0003-4194-2574 \\ María Isabel Vidal-Esteve*** iD orcid.org/0000-0002-3504-8114 \\ María Monserrat Castro-Rodríguez**** iD orcid.org/0000-0002-5544-4421
}

\section{Para citar este artículo}

López-Gómez, S., Rodríguez-Rodríguez, J., Vidal-Esteve, M. I. y Castro-Rodríguez, M. M. Contribuciones y efectos de los videojuegos en la atención a la diversidad. Revista Colombiana de Educación, 1(84), 1-25.

https://doi.org/10.17227/rce.num84-12742

\section{(ㄷ) $(1) \Theta$}

* Profesora del Departamento de Pedagogía y Didáctica, Universidad Santiago de Compostela, Galicia, España. Correo: silvialopez.gomez@usc.es

* Licenciado en Pedagogía (Didáctica y Organización Escolar), Universidad Santiago de Compostela, Galicia, España. Correo: jesus.rodriguez.rodriguez@usc.es

*** Investigadora en Didàctica i Organització Escolar, Universitat de València, Comunidad valenciana, España. Correo: Isabel.Vidal@uv.es

**** Profesora del Departamento de Pedagogía y Didáctica, Universidade da Coruña, A Coruna, Galicia, España. Correo: maria.castror@udc.es 


\author{
Palabras clave: videojuegos; \\ educación; atención a la \\ diversidad; educación \\ inclusiva
}

Keywords: video games; education; attention to diversity; inclusive education

\section{Resumen}

Este artículo presenta los resultados de una revisión de la literatura sobre estudios centrados en las contribuciones y los efectos de los videojuegos en la atención a la diversidad. Para ello, se tomó como muestra las investigaciones recogidas en las bases de datos Scopus, wos, eric, ProQuest Dissertations \& Theses Global (pqdt), Dialnet y csicisoc y en los buscadores académicos Rebiun, Teseo y ScienceDirect. Después de considerar algunos aspectos del análisis bibliométrico, con el propósito de contextualizar la autoría y la procedencia de los artículos, se procedió a analizar en profundidad el contenido de los documentos seleccionados, de acuerdo con los objetivos de este estudio. Fruto de la interpretación del contenido se elaboró una clasificación categorial que refleja las líneas de investigación en el campo de los estudios sobre videojuegos y educación inclusiva. Las cuatro grandes categorías sobre los efectos de los videojuegos en la atención a la diversidad se relacionan con: alumnado con diversidad funcional y trastornos, posibles efectos perjudiciales ante altos niveles de uso de videojuegos, efectos sobre el aprendizaje escolar e influencia de los videojuegos sobre las habilidades procedimentales y actitudinales. Los resultados evidencian la necesidad de favorecer la adquisición de competencias digitales, el desarrollo emocional de los jugadores y jugadoras, y la prioridad de profundizar en la creación de videojuegos que favorezcan la educación inclusiva.

\section{Abstract}

This article presents the results of a literature review of studies focused on the contributions and effects of video games in the attention to diversity. For this purpose, research collected in the Scopus, wos, eric, ProQuest Dissertations \& Theses Global (pqdt), Dialnet and csic-isoc databases and in the academic search engines Rebuin, Teseo and ScienceDirect were taken as a sample. After considering some aspects of the bibliometric analysis in order to contextualize the authorship and origin of the articles, we proceeded to conduct an in-depth analysis of the content of the selected documents, in accordance with the objectives of this study. As a result of the interpretation of the content, a categorical classification was elaborated that reflects the lines of research in the field of studies on videogames and inclusive education. The four main categories on the effects of video games on attention to diversity are related to: students with functional diversity and disorders, possible detrimental effects of high levels of video game use, effects on school learning and the influence of video games on procedural and attitudinal skills. The results show the need to promote the acquisition of digital skills, the emotional development of players, and the priority of deepening the creation of video games that promote inclusive education.

\section{Resumo}

Este artigo apresenta os resultados de uma revisão da literatura sobre estudos focados nas contribuições e efeitos dos videogames na atenção à diversidade. Para tanto, foram utilizadas as pesquisas coletadas nas bases de dados Scopus, wos, eric, ProQuest Dissertations \& Theses Global (pqdt), Dialnet e csic-isoc e nos buscadores acadêmicos Rebuin, Teseo e ScienceDirect. Após considerar alguns aspectos da análise bibliométrica para contextualizar a autoria e a origem dos artigos, foi realizada uma análise aprofundada do conteúdo dos documentos selecionados, de acordo com os objetivos deste estudo. Como resultado da interpretação do conteúdo, foi desenvolvida uma classificação categórica que reflete as linhas de pesquisa no campo dos estudos de videogame e educação inclusiva. As quatro categorias principais sobre os efeitos dos videogames na atenção à diversidade estão relacionadas a: alunos com diversidade e distúrbios funcionais, possíveis efeitos nocivos do alto nível de uso de videogames, efeitos na aprendizagem escolar e a influência dos videogames nos alunos e habilidades atitudinais. Os resultados mostram a necessidade de favorecer a aquisição de competências digitais, o desenvolvimento emocional dos jogadores e jogadoras e a prioridade de aprofundar a criação de videojogos que favoreçam a educação inclusiva. 


\section{Introducción}

Las tecnologías de la información y la comunicación (TIC) están incorporándose a las escuelas con esperanzas de cambio en pro de la superación de obstáculos tales como el absentismo o el abandono escolar, y también como respuesta a las necesidades educativas del alumnado.

Uno de los motivos de la implementación de las TIC en el ámbito educativo apunta a las oportunidades a nivel didáctico e inclusivo que ofrece (Reyes y Prado, 2020; Rodríguez y Arroyo, 2014), y otra razón, es que se han convertido en "una de las principales y más destacadas señas de identidad de los jóvenes, la denominada generación de los millennials" (Area et al., 2015, p. 13). En este contexto en el que su uso se incrementa exponencialmente, una de las vertientes más novedosas e innovadoras es el empleo de videojuegos en las aulas.

El juego ha sido un recurso didáctico empleado a lo largo de la historia por ser considerado uno de los elementos clave para la construcción de pensamientos y comportamientos, según la teoría constructivista de Piaget (Saldarriaga et al., 2016), o como un elemento fuertemente motivacional y de interés para la adquisición de conocimiento, según la concepción del aprendizaje significativo de Ausubel (Sampedro, 2012). Y con la irrupción de las tecnologías, este principio didáctico ha desembocado en los juegos digitales y en los videojuegos, que pueden ser empleados con funcionalidades similares (Marín y Ramírez, 2012), tales como el desarrollo del pensamiento reflexivo, de las capacidades de atención y memoria, de habilidades para resolver conflictos, entre otros (Santos et al., 2014).

Mucho se ha escrito, en los últimos años, sobre los usos y beneficios de los videojuegos y de la gamificación en todos los niveles educativos (González, 2020; Holguin et al., 2020), y respecto a sus potencialidades con alumnado con diversidad, pese a que el número de estudios es mucho menor (Cabero y Ruiz, 2017), se registran investigaciones tanto en aulas inclusivas y en el hogar, como en centros específicos y en entornos hospitalarios (Mejía et al., 2019) obteniéndose beneficios en la comunicación, la atención, la planificación cognitiva, la memoria y la autonomía, entre otros (Baldassarri et al., 2020; Vidal et al., 2017).

En el Estado español, el alumnado con necesidades específicas de apoyo educativo (NEAE) es, según la Ley orgánica 8/2013, de 9 de diciembre, para la mejora de la calidad educativa (LOMCE), aquel que requiere, de forma temporal o permanente, una atención educativa diferente a la ordinaria por presentar necesidades educativas especiales, dificultades específicas de aprendizaje, altas capacidades intelectuales, trastorno por déficit de atención e hiperactividad (TDAH), por incorporarse tardíamente al sistema educativo o por condiciones personales o de historia escolar. Las tecnologías, en sus distintos usos y aplicaciones, constituyen una valiosa herramienta para el desarrollo de la educación inclusiva, ya que permiten hacer viables los principios de "acceso, calidad, igualdad, justicia social, democracia, participación, buscando el equilibrio entre comunidad y diversidad" (Lázaro et al., 2015, p. 5). Según Sampedro y McMullin (2015), encontraremos un aliado en cualquier tipo de herramienta que beneficie la adquisición de conductas y conocimientos; y los videojuegos son un claro ejemplo de ello dado su carácter motivador y lúdico, que además "propicia la socialización, la equidad y la igualdad, elimina barreras producidas por cualquier tipología de 
exclusión [...] y, concretamente, favorece los principios de presencia, participación y progreso" (p. 129).

Sin embargo, en esta investigación no nos ceñimos al concepto de inclusión centrada en la discapacidad ni en el alumnado con NEAE, sino que se toma en consideración la definición que, desde la perspectiva del DUA (diseño universal de aprendizaje), emplea en España, la Ley orgánica 3/2020, de 29 de diciembre, por la que se modifica la Ley orgánica 2/2006, de 3 de mayo, de educación (LOMLOE) sobre la atención a la diversidad entendida como el conjunto de actuaciones educativas dirigidas a dar respuesta a las diferentes capacidades, ritmos y estilos de aprendizaje, motivaciones, intereses, situaciones socioeconómicas y culturales, lingüísticas y de salud del alumnado.

Según algunos estudios, los videojuegos se convierten en recursos eficaces para la inclusión en las aulas, considerando que propician el desarrollo de competencias, entre las que destaca la competencia social y cívica. Esta, según Pagès (2009), puede considerarse como la principal para la enseñanza ya que, la finalidad fundamental del sistema educativo no es otra que formar ciudadanas y ciudadanos que sepan convivir democráticamente con los demás, participando en todas las esferas de la vida e intentando mejorarla. Así pues, contribuir al desarrollo de la competencia social y cívica, como lo hace el juego y su alternativa digital, los videojuegos, supone implícitamente favorecer a la aplicación y adquisición de una cultura inclusiva (Puig y Morales, 2015). Igualmente, las propias características de los videojuegos pueden favorecer el uso de juegos por parte de personas con capacidades muy diferentes: organización por niveles, progresión en las habilidades exigidas, etc. Así pues, tal y como señalan Romera et al. (2008) y Rodríguez y Arroyo (2014), los videojuegos, como recursos innovadores, eficaces y significativos para el proceso de enseñanza y aprendizaje de todos, son además una herramienta didáctica eficaz para desarrollar todos los planos de la inclusión que repercuten en la sociedad a través del fomento de la competencia social y cívica.

Partiendo de este marco contextual, consideramos necesario llevar a cabo una revisión del estado del arte sobre las aportaciones de los videojuegos al desarrollo de una escuela inclusiva que considere la diversidad como motor de cambio para la sociedad y el aprendizaje, y que permita verificar si verdaderamente posibilita, como apuntan algunos autores, el acceso al aprendizaje sin el prejuicio de la exclusión (Earp et al., 2014).

\section{Metodología}

En esta investigación se realiza una revisión de la literatura sobre los estudios empíricos desarrollados alrededor de los videojuegos y la educación inclusiva desde dos vertientes. Por una parte, desde la perspectiva cienciométrica, se llevó a cabo un análisis bibliométrico parcial para aportar datos iniciales sobre la actividad científica y la situación del objeto de estudio (Tomás-Górriz y Tomás-Casterá, 2018). En concreto, los indicadores analizados se agrupan en torno al tipo de publicación, el número de documentos por año, el idioma, los países punteros, las autorías más relevantes en el campo y los términos más frecuentes. En segundo lugar, se desarrolló el análisis del contenido de los datos bibliográficos en torno a cuatro categorías que surgieron de los resultados. Así pues, el objetivo principal consistió en identificar las principales líneas de investigación desarrolladas en este campo, prestando especial atención a las publicaciones generadas en los últimos años. 
Para ello, se tomó como muestra de este estudio las investigaciones recogidas en las bases de datos Scopus, wOS, ERIC, ProQuest Dissertations \& Theses Global (PQDT), Dialnet y CSICISOC y en los buscadores académicos Rebiun, Teseo y ScienceDirect.

Para la búsqueda bibliográfica, no se realizó restricción temporal en cuanto a fecha de publicación, siendo empleados los siguientes términos de búsqueda: "serious games", "video games", "videogames", "videojuegos" combinados en algunos de los casos con "education" o "educación", "diversity" o "diversidad" e "inclusive education", "inclusion" o "educación inclusiva" e "inclusión". Las búsquedas se adaptaron a los operadores booleanos y criterios de búsqueda de las bases de datos y buscadores consultados.

Aunque la búsqueda no se limitó temporalmente, en los últimos diez años surge una elevada proliferación de la investigación sobre el ámbito de los videojuegos relacionados con la visibilización de la atención a la diversidad. Además, se fusionaron los resultados obtenidos a través de las distintas bases de datos y buscadores académicos y se suprimieron las duplicidades. Queda destacar que también se incorporaron algunos documentos que se consideraron pertinentes para nuestro tema objeto de estudio, a través de la búsqueda inversa; "un método de selección de documentos realizado a partir de la bibliografía de los documentos primarios recuperados en la búsqueda" (Gálvez, 2002, p. 6).

Para el desarrollo de esta investigación se realizó una búsqueda sistemática en el mes marzo de 2021 obteniendo un total de 769 documentos (disponibles en https://go.uv.es/1nkFeRZ). No obstante, teniendo en cuenta que nuestro objeto de estudio se centró específicamente en el ámbito de la atención a la diversidad, de esta búsqueda, se seleccionaron aquellos estrictamente relacionados con el tema; un total de 319 documentos.

Para la selección de los artículos del análisis de contenido, se hizo una primera lectura de sus títulos y resúmenes, seleccionando los considerados relevantes para su revisión a texto completo y excluyendo los de difícil acceso o no disponibles de forma gratuita, y aquellos cuyos resultados no tuvieran en consideración directamente la atención a la diversidad. Con el propósito de analizar las investigaciones, se tuvieron en cuenta los siguientes aspectos: autoría, año de publicación, tipología de documento (tesis, artículo, comunicación, informe...), idioma, título, objetivo principal del estudio, método, muestra (número y características) y principales resultados.

Después de analizar el contenido de los documentos seleccionados, de acuerdo con los objetivos de este estudio, se elaboró una clasificación categorial que refleja las líneas de investigación en el campo de los estudios sobre videojuegos y educación inclusiva. Estas investigaciones se han clasificado en torno a cuatro grandes categorías: efectos de los videojuegos sobre el alumnado con diversidad funcional y trastornos, posibles efectos perjudiciales ante altos niveles de uso de videojuegos, efectos sobre el aprendizaje escolar e influencia de los videojuegos sobre las habilidades procedimentales y actitudinales. Estas serán presentadas en el siguiente apartado, tras los datos bibliométricos que permitirán contextualizar el estado del arte, en base a los rasgos cuantificables extraídos de la literatura científica. 


\section{Resultados}

A continuación, presentamos, en primer lugar, los resultados de algunos aspectos del análisis bibliométrico considerados con el propósito de contextualizar la autoría y la procedencia de los artículos.

Por un parte, en relación con el tipo de publicación, de los 769 documentos, la mayoría son comunicaciones $(n=384)$, precedidos por artículos $(n=279)$ y capítulos de libro $(n=40)$. En cuanto al idioma, destaca el inglés con 734 documentos frente al español con únicamente 21 documentos, el portugués $(n=9)$, el francés $(n=1)$ y el alemán $(n=1)$.

En cuanto al número de documentos por país, es visible la desigualdad en la producción científica en revistas de impacto, despuntando Estados Unidos (con 179 documentos) seguido por España $(n=87)$, Reino Unido $(n=68)$, Italia $(n=51)$ y Grecia $(n=39)$. Es de remarcar que entre los países iberoamericanos se contemplan Colombia (con 12 documentos), Chile ( $n$ $=9)$, Ecuador $(n=6)$, Perú $(n=6)$, México $(n=5)$ y Argentina $(n=5)$, que suponen en total un $5,6 \%$ de la producción total.

En referencia a las autorías más prolíficas, se distinguen Meyer, Arnab y Brom, con 9, 7 y 6 documentos, respectivamente. Y entre las universidades con más publicaciones destacan la Coventry University $(n=9)$, Università degli Studi di Genova $(n=8)$ y The University of Hong Kong, University of Central Florida y la Universidad de Granada, con 7 documentos cada una.

En cuanto al número de documentos por área, es de destacar que la mayoría de los textos, $34 \%(n=489)$, se enmarcan en las Ciencias Computacionales, seguido por $26,4 \%(n=380)$ de las Ciencias Sociales, la Ingeniería (13,8 \%), las Matemáticas (5,1\%) o la Medicina (5 \%).

En cuanto a la fecha de publicación, es importante remarcar que, a partir de 2013, el número de publicaciones por año aumentó de forma significativa, superando los 50 documentos por año, hasta 2017 donde se observa su máximo relativo. Aún así, se observan pequeñas regresiones posteriormente, como se observa en la figura 1. No obstante, podemos considerar que la tendencia fue el aumento de este tipo de publicaciones científicas. 


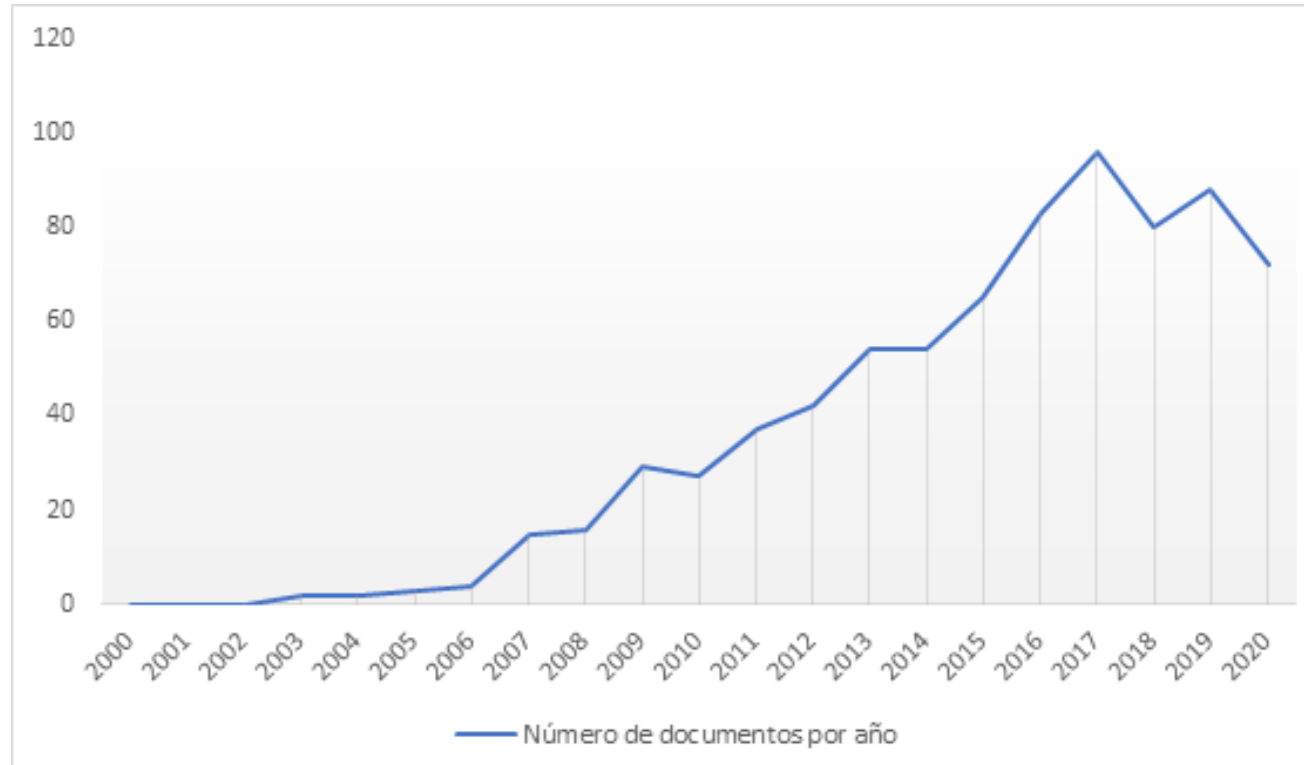

Figura 1. Distribución de los documentos por fecha de publicación Fuente: elaboración propia a partir de los datos obtenidos.

Por lo que se refiere a la repercusión e impacto de los artículos analizados, se extrae que un total de 5473 documentos citan estas investigaciones, destacando como las publicaciones más citadas las de Annetta et al. (2009) con 355 citas, seguido por Bourgonjon et al. (2010), Gros (2007) y Shaffer (2006), todas ellas con más de 200 citas.

Por último, en cuanto al contenido, es posible analizar a través de la herramienta vosViewer, las palabras clave más frecuentes, así como su agrupación en clústeres o nodos relacionales. Como se puede comprobar en la figura 2, destaca la elevada cantidad y diversidad de términos, 46 en total, con más de 30 coocurrencias, que se agrupan en torno a 5 nodos. Entre los términos destaca principalmente child como agente clave en el proceso, video game como objeto central de nuestro análisis y technology como contexto y medio en el que se ubica lo analizado. Además, son de remarcar términos como motivación, enseñanza, actitudes, aprendizaje, juego, potencial, reto o capacidades, que están estrechamente relacionados con la perspectiva inclusiva de atención a la diversidad desde la que partía este estudio. 


\section{\& VOSviewer}

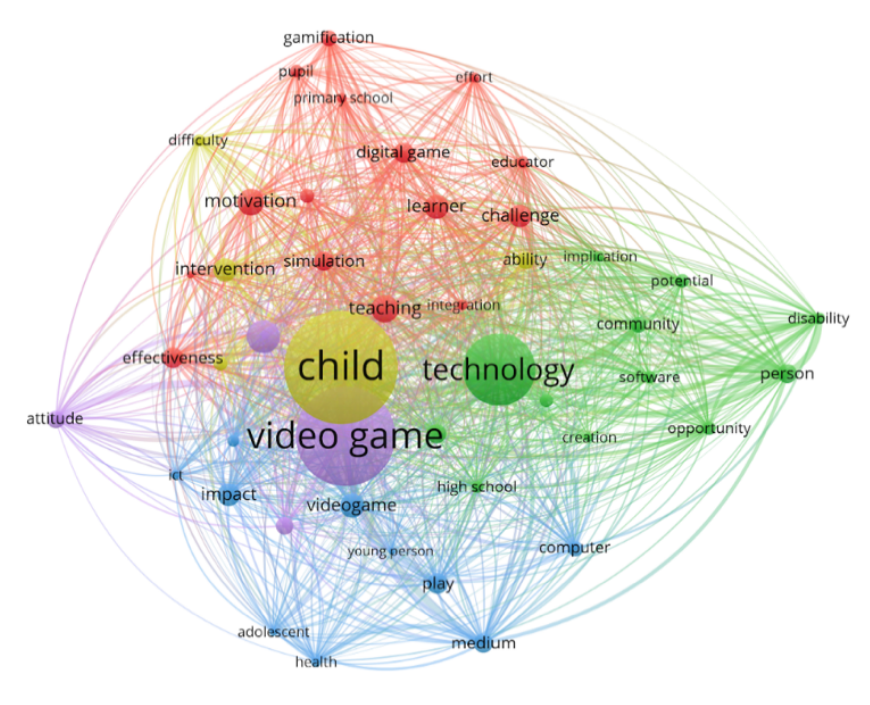

Figura 2. Mapa de coocurrencia de términos Fuente: vosViewer.

A partir de estos datos bibliométricos que contribuyen a contextualizar el estado del arte sobre los videojuegos y la atención a la diversidad, procedemos a profundizar en el análisis de contenido de los 319 documentos seleccionados, de acuerdo con las cuatro categorías previamente mencionadas que relacionan los videojuegos con el alumnado con diversidad funcional y trastornos, sus posibles efectos adversos, el aprendizaje y su influencia sobre las habilidades procedimentales y actitudinales.

\section{Alumnado con diversidad funcional y trastornos}

Este tipo de estudios enfatizan en el impacto que ejercen los videojuegos comerciales sobre las funciones cognitivas (memoria y funciones ejecutivas), participación en el aula y atención. García-Redondo et al. (2019) afirman que los videojuegos están siendo muy utilizados en alumnado con NEAE, especialmente para mejorar su bienestar, las habilidades sociales, la vida independiente de las personas con trastorno del espectro autista (TEA), con discapacidad intelectual o con altas capacidades. Por ejemplo, en Armendarez (2015), Rodríguez-Jiménez (2015), Hillier (2013) o Sproull (2011), se exponen los resultados de investigaciones en las que participan fundamentalmente niñas y niños con TEA, con síndrome de Down, con trastornos específicos del lenguaje (TEL), y déficit de atención e hiperactividad (TDAH). En este sentido, el estudio de Armendarez (2015) examina el uso de los videojuegos en infantes y adolescentes con TDAH de Rhode Island (Estados Unidos) a partir de casos clínicos, comparando los resultados de uso con los datos nacionales. Los 131 padres y madres son los que proporcionan los datos cumplimentando una encuesta de 13 cuestiones. El estudio concluye que los niños juegan más a los videojuegos que las niñas, y los jóvenes de mayor edad (11-14 años) más que los que cuentan con una inferior (5-7 años). La mayoría de los padres y madres indican que, durante el tiempo de juego, sus hijos e hijas experimentan 
disminución de los comportamientos asociados al TDAH, es decir, menor falta de atención, menor hiperactividad e impulsividad y menor desorganización.

En el mismo año, y siguiendo con las investigaciones que emplean videojuegos comerciales, el objetivo principal de la tesis de Rodríguez-Jiménez (2015) es el entrenamiento en las funciones ejecutivas y de la memoria en personas con NEAE. Para eso, lleva a cabo un estudio piloto y tres experimentos independientes, en los que participan niños y adolescentes con síndrome de Down y con otras necesidades educativas especiales (NEE). En cuanto a los resultados, se observa una mejora en algunos de los aspectos analizados, sobre todo los relacionados con la memoria. Ren y Wu (2019), después de una revisión del efecto rehabilitador de la realidad aumentada (RA) con niños y niñas con parálisis cerebral, donde hay efectos positivos en el desarrollo de la motricidad gruesa, llegan a la conclusión de la necesidad de aumentar las muestras y la calidad de los ensayos.

En este mismo año, Ramos-Ramírez y Mauricio (2019), en una reducida muestra de 10 menores sordomudos, utilizan un videojuego orientado al procesamiento de imágenes para la enseñanza del español. Tras su uso, se registra un incremento en cuanto al aprendizaje y en sus calificaciones de un $22 \%$ respecto al inicio.

En general, las investigaciones atribuyen a los videojuegos efectos muy positivos en el desarrollo de distintas competencias en el alumnado con diversidad funcional. Sin embargo, algunas otras han introducido importantes cuestionamientos que rompen con la brecha divisoria entre personas con diversidad y las consideradas de desarrollo típico. Por ejemplo, Cho et al. (2017) constatan que adolescentes con TEA coinciden con adolescentes con desarrollo típico y muestran intereses muy semejantes en cuanto a los videojuegos.

Por su parte, Kaigo y Okura (2020) realizan una interesante investigación que tiene como objetivo analizar cómo y qué criterios tienen en consideración las empresas que elaboran videojuegos para personas con discapacidad motriz en Japón. Los resultados son muy interesantes: introducen herramientas para el manejo fácil, recursos para el apoyo a personas con discapacidad visual basándose en dos aspectos: el concepto de tecnología de asistencia especializada o en la reducción de barreras. Atribuyen especial relevancia a la necesidad de favorecer la participación social de las personas con este tipo de diversidad.

El éxito de la implementación de los videojuegos también pone en evidencia la complejidad de la intervención en personas con diversidad funcional o trastornos, debido a la variedad de características que se puede identificar en una misma diversidad. La investigación desarrollada por Sefen et al. (2020) analiza el uso de videojuegos que favorecen la actividad física en personas con TEA y exige la condición de adaptabilidad a las condiciones personales individuales.

En esta subcategoría también deben ser citados los trabajos dirigidos a diseñar y analizar la eficacia de videojuegos específicos o inclusivos. Se trata de estudios de viabilidad para determinar los beneficios o mejoras a implementar en los videojuegos diseñados para personas con discapacidad. Son los trabajos de Jaume et al. (2013); Gerling et al. (2015); Lyon (2015); Tobar et al. (2015); Corrales-Astorgano et al. (2019); entre otros.

Entre los más recientes destaca la investigación de Lyon (2015), en la que diseña el juego digital Feeling Factory, con el objetivo de mejorar la prosodia en niños(as) con TEA. La autora estudia su viabilidad a través de entrevistas semiestructuradas con un panel de expertos y 
con cinco niños con TEA de entre 12 a 14 años. El estudio concluye con resultados positivos, entre los que se destaca la utilidad y eficacia del juego. Resultados muy similares son los de Corrales-Astorgano et al. (2019), en cuanto al uso de un videojuego para el trabajo de la prosodia en niñas y niños afectados por síndrome de Down.

Por su parte, Tobar et al. (2015) diseñan un videojuego inclusivo con realidad aumentada (RA) para el aprendizaje de habilidades de lógica matemática. Prueban el juego con un conjunto de 20 estudiantes con diversas NEAE, mostrando que el desempeño durante el juego es similar en niños y niñas. Se concluye que la RA y el aprendizaje basado en juegos digitales permiten la integración del alumnado con NEAE en el proceso formativo.

Conviene indicar que existen trabajos que dirigen su mirada al consumo que de estos medios realizan las personas con diversidad, como el estudio de Lozano et al. (2014), en el que presentan los resultados de una investigación en 15 centros educativos de la Comunidad Autónoma de la Región de Murcia (España). En ella, se pretende conocer, a través de un cuestionario, el equipamiento, uso y consumo de medios digitales por parte del alumnado con NEAE de 30 y 40 de educación secundaria obligatoria (TESO), así como la existencia de diferencias respecto al alumnado sin dichas necesidades. Para eso, obtienen una muestra de 2734 alumnos y alumnas, de los que 487 tenían NEAE. En cuanto al tipo de juego, se comprueba que los preferidos son los de deportes, coches y carreras $(38,7 \%)$ constatándose diferencias con respeto al grupo sin NEAE, lo cual optó por los de karaoke, concursos... (29,7 \%). En la investigación también se constata que en los hogares del alumnado con NEAE hay menos equipamientos informáticos (como ordenadores y acceso a la red) que en el resto de la muestra. Se indica que no prestan tanta importancia a los estudios y a las amistades, prefiriendo restarles tiempo para conectarse a la red, ver la televisión y utilizar las redes sociales.

En los últimos años también han aparecido investigaciones centradas en el uso de videojuegos con personas con distintas diversidades y trastornos con relación a las distintas materias o competencias del currículum, tal y como se muestran a continuación.

Franceschini et al. (2017) realizan una investigación centrada en analizar el impacto de otro programa orientado para niños y niñas angloparlantes afectados por la dislexia, obteniéndose resultados muy positivos en la mejora de la atención visoespacial, memoria de trabajo fonológica, aceleración de la atención visual, la velocidad de reconocimiento y decodificación fonológica, etc.

Desde la comunidad educativa, la percepción es distinta. Por ejemplo, Dos SantosVenturelli y Denise-Pereira (2019) realizan una investigación con profesorado, donde se les pregunta sobre la relevancia del uso de videojuegos en el ámbito de las matemáticas con alumnado con discapacidad intelectual. Los resultados reflejan el optimismo que los docentes atribuyen al uso de videojuegos en el éxito académico, aunque asumen la falta de formación profesional para abordar este reto. Contrariamente, Jasem y Delport (2019) en una investigación realizada con mujeres que tienen hijos e hijas con TDAH, asumen el gran consumo de videojuegos durante horas, pero no les atribuyen a estos recursos beneficios para el desarrollo cognitivo.

Por su parte, y a modo de ejemplo en el contexto hospitalario, Guerra y Revuelta (2015) describen la propuesta metodológica para las aulas hospitalarias de Extremadura, utilizando el videojuego Minecraft. Desarrollan una metodología educativa basada en el uso de 
videojuegos como herramienta pedagógica dentro del contexto hospitalario. Inciden en el plano emocional para establecer contextos emocionales positivos que fomenten el aprendizaje, la cooperación, la interacción social y la motivación a través del uso de videojuegos y que además funcionen como complemento al currículo común.

\section{Posibles efectos perjudiciales ante altos niveles de uso de videojuegos}

A continuación, se presenta una muestra de posibles efectos adversos asociados a niveles elevados de uso de videojuegos y la influencia en la conducta que pueden llegar a ejercer sobre la diversidad de los usuarios. En algunos casos los efectos pueden ser tan graves que se llegan a asociar con trastornos mentales, emocionales o atencionales.

\section{Adic ción}

En el estudio de Demirtas et al. (2015), en el que se analizan las tasas de adicción a los videojuegos de 396 estudiantes de educación primaria de la ciudad de Ankara (Turquía), los resultados indican que las tasas de adicción a los videojuegos de los niños son más altas que las de las niñas, pero no encontraron diferencias significativas con respecto a la tasa de adicción y nivel educativo familiar.

Liu (2014) va un paso más allá y realiza un estudio longitudinal en el que analiza los datos conseguidos en una serie de estudios sobre la salud de los adolescentes de Estados Unidos, en los tramos 1994-1995, 1996, 2001-2002. Los resultados ofrecen un panorama mixto en el que surgieron tanto efectos negativos como positivos dispares al jugar a videojuegos más de 21 horas a la semana. La autora del estudio sugiere que es necesaria más investigación para determinar la naturaleza exacta y las consecuencias de la adicción a los videojuegos. Resalta que, a pesar de obtener resultados positivos para la salud asociados con un uso abusivo de los videojuegos, conviene limitar el uso de estos medios a menos de 3 horas por día durante los años formativos de la adolescencia, y así reducir el riesgo de resultados negativos a largo plazo, como la deserción escolar y la depresión.

\section{Violencia}

Algunas investigaciones vinculan la violencia con los videojuegos en dos dimensiones opuestas. Por una parte, existen estudios que resaltan que algunos videojuegos promueven o favorecen actitudes violentas y conductas agresivas en los usuarios, tal y como afirma Ángel (2020). Sin embargo, otros videojuegos se conciben para trabajar con personas que muestran conductas agresivas o para la adquisición de competencias transversales, como el autocontrol o la toma de decisiones para mejorar las relaciones interpersonales.

En este sentido, Reyes-Hernández et al. (2014) identifican entre las posibles aportaciones de algunos videojuegos, la generación de un clima de relajación y distensión, con efectos muy positivos en la conducta de ciertas personas. Resultados semejantes lo constatan ReyesPadilla et al. (2020). Kneifer (2014) realiza una investigación de corte cualitativo de grupos focales en la que se examinan los efectos nocivos de los videojuegos violentos sobre las 
emociones y la conducta. Se comparan las respuestas de alumnado universitario, entre los que se encuentran jugadores(as) de esta tipología de medio. Los resultados apoyan la presencia, así como la ausencia de efectos violentos en la conducta de estos(as) videojugadores(as).

Por el contrario, trabajos como el de Cusmano (2010) destacan especialmente las respuestas de ira y agresión ante la exposición a determinados videojuegos violentos.

Investigaciones posteriores como de Papa Gusho y Mitrushi (2017) recalcan la relación entre el uso de videojuegos violentos y las conductas desarrolladas posteriormente por los (las) adolescentes, que, con frecuencia, suelen traducirse en conductas y actitudes también violentas.

\section{Estrés, ansiedad y depresión}

Examinan la relación entre el tiempo en pantalla y los síntomas de los trastornos emocionales complejos. Son los trabajos de Brom et al. (2014) o Maras et al. (2015). Este último equipo de investigación examina la relación entre el tiempo de pantalla y los síntomas de depresión y ansiedad en una muestra de 2482 jóvenes canadienses. Se utilizan datos transversales recopilados entre 2006 y 2010 como parte de una investigación sobre los estilos de vida de la juventud (en concreto: Research on eating and adolescent lifestyles [REAL]). En los resultados se destaca que la duración del tiempo en pantalla jugando a videojuegos puede asociarse con la severidad de la depresión y la ansiedad en adolescentes. Indican, al igual que la mayoría de los trabajos que analizan los efectos negativos de los videojuegos, que son necesarias pesquisas adicionales para determinar si la reducción de tiempo de juego ayuda a la prevención y el tratamiento de estos trastornos psiquiátricos en la juventud.

Se incorpora en esta línea el estudio de Kasper et al. (1999), en el que se exploran los posibles problemas cardiovasculares que pueden llevar asociados un uso excesivo de videojuegos. Además, Guerrero y Valero (2013) estudian los efectos secundarios tras el uso de la realidad virtual (RV) en un videojuego, entre los que se encuentran malestar y ansiedad, efectos sobre el equilibrio, problemas de atención, entre otros.

Mathieu et al. (2020) estudian los factores asociados a cada tipo de juego, su intensidad, los motivos del juego y las distorsiones cognitivas en 291 jugadores masculinos con distinto grado de habilidad para el juego. Según los resultados, la selección de videojuegos debe estar ligada a su tipología de juego, la angustia psicológica, los motivos de juego y las distorsiones cognitivas que presenta cada persona.

López-Rodríguez et al. (2020) adelantan una revisión sistemática de los efectos de los videojuegos en niñas y niños enfermos de cáncer. Su conclusión es que se han obtenido herramientas positivas para la reducción de la ansiedad y la sensación del dolor.

Algunos artículos hacen hincapié en la necesidad de realizar investigaciones longitudinales extensas sobre el impacto del videojuego en las personas que presentan NEAE derivadas de trastornos, con el objetivo de constatar o refutar resultados de otros trabajos y que permiten extraer conclusiones más consolidadas (Wartberg et al., 2020). 


\section{Falta de atención en el aula}

Si bien, como ya se indicó a lo largo de este artículo, existen investigaciones en las que se corrobora un aumento de la motivación y atención del alumnado cuando se emplean videojuegos como recursos en el aula, se encuentran otros estudios, como el de Wallace (2012), en el que se evidencia una relación positiva significativa entre la cantidad de horas que los infantes pasan jugando a videojuegos, sobre todo los clasificados como de acción, y la falta de atención e hiperactividad en las aulas.

En general, en los resultados de estos estudios se aconseja no jugar a videojuegos durante periodos prolongados de tiempo. En consonancia resaltan la necesidad de investigaciones adicionales que permitan dilucidar los posibles efectos negativos asociados a un mal uso de los videojuegos.

\section{Aprendizaje}

Distintas corrientes pedagógicas o metodológicas inciden en la necesidad de dar respuestas a todo el alumnado que está escolarizado en un aula o escuela, sean cuales sean sus condiciones y características personales. La producción y la investigación en torno a los videojuegos no ha quedado al margen de este contexto, y proliferan las líneas de investigación más representativas en este ámbito; las que indagan en el impacto de los videojuegos en el aprendizaje, y en los efectos que ejercen en las habilidades procedimentales y actitudinales del alumnado. En los últimos años, también se entiende cómo estos recursos pueden contribuir de forma positiva al aprendizaje del alumnado con condiciones personales que no tienen una presencia mayoritaria en los centros escolares.

Cipollone (2015) emplea el conocido videojuego Minecraft, para valorar en un grupo de alumnos y alumnas sus niveles de motivación intrínseca. La motivación se erige como una variable que contribuye a la mejor predisposición para aprender y a la adquisición de estrategias para la memorización o la resolución de problemas. Los resultados muestran la existencia de elevados niveles de motivación cuando los estudiantes empiezan a ser capaces de controlar el juego, lo que contribuye a su inmersión en el entorno del videojuego. Por su parte, aunque bien es cierto que no se observan modificaciones en algunas habilidades relacionadas con el aprendizaje, como la memorización de definiciones, el uso de Minecraft contribuye positivamente, en este caso, a otras habilidades como la resolución de problemas.

En el estudio de Zhao y Linaza (2015), por su parte, participan estudiantes de los cursos de $20,4^{\circ}$ y $6^{\circ}$ de educación primaria (siguiendo el sistema educativo español), con el fin de conocer qué habilidades se emplean y qué resultados de aprendizaje se obtienen mediante el uso de un nuevo videojuego. De esta investigación surge la contribución en numerosas áreas como las habilidades de resolución de conflictos en grupo, la mejora de la responsabilidad y de la autonomía en el aprendizaje, sin intervención docente, en alumnado de todos los cursos, y las habilidades para construir significados sobre el espacio y el contexto virtual del videojuego. Además, en los jugadores de menor edad, surgen habilidades complejas y 
fundamentales para el juego, como el cuidado de los(as) compañeros(as), el control del proceso y la capacidad de liderazgo.

Navaneetham y Chandran (2018), con una muestra de 200 estudiantes de los últimos cursos de la escolarización obligatoria, indagan la relación entre el consumo de videojuegos y su impacto en el aprendizaje escolar. Se obtienen resultados distintos a los de otras investigaciones, ya que se considera que no hay diferencias en los hábitos de estudio entre ambos sexos. Esto conduce a estos autores a afirmar que la percepción, la actitud hacia las tareas académicas, así como los objetivos son también agentes claves en el aprendizaje.

La investigación de Contreras et al. (2019) realizada con 34 estudiantes con discapacidad intelectual escolarizados en educación primaria y secundaria, concluye que la selección y uso de videojuegos adaptados a cada estudiante, puede incidir positivamente en las habilidades de coordinación, de ejecución del movimiento, conciencia corporal, fomentar la atención, la planificación y las habilidades de aprendizaje.

\section{Influencia de los videojuegos sobre las habilidades procedimentales y actitudinales}

A continuación, se presentan las características de los estudios relacionados con esta línea:

\section{Comunidades de práctica}

Bell y Gresalfi (2017), en un estudio de caso con una profesora que trabaja con 61 estudiantes de 70 procedentes de grupos vulnerables, donde utilizan el videojuego Boone's Meadow, concluyen que el éxito en los procesos de integración social y utilidad está en relación con la experiencia de los docentes en el uso del videojuego.

\section{Construcción de la identidad}

Cortés et al. (2012) recogen en un artículo los resultados de una investigación realizada en tres céntricos institutos madrileños de educación secundaria, donde se lleva a cabo un proyecto de intervención, en el que se crea una red social "Jugar y Aprender", en la que se utiliza videojuegos comerciales como Los Sims 3. Los resultados visibilizan la contribución de ambas herramientas a la construcción de la identidad (participación, colaboración, solución de problemas...). El éxito en la consecución de los objetivos del proyecto se le atribuye no solo al cómo utilizar el videojuego y las redes sociales, sino a las sinergias y a las relaciones interpersonales y comunicativas que emergen cuando el alumnado está viviendo y comprendiendo la historia del videojuego. 
Sociabilidad

María Monserrat Castro-Rodríguez

Una línea muy interesante de investigación en el ámbito de los videojuegos es su contribución a la sociabilidad. El trabajo realizado por Uz y Cagiltay (2015) en Turquía, en su momento, introduce relevantes aportaciones, en tanto que sus hallazgos generan nuevas líneas de investigación, debido a que se ha cuestionado la idea de que la práctica de los videojuegos sea un obstáculo para establecer relaciones personales en la vida real. De hecho, según sus resultados, la mayoría de jugadores de videojuegos prefieren jugar con amistades y familiares de la vida real. Como conclusión también destacan que no pueden confirmar los resultados de estudios precedentes en los que se le atribuía a las personas tímidas o introvertidas dificultades para entablar relaciones sociales en la vida real.

\section{Resolución de problemas de forma colaborativa}

En la literatura revisada, también se encuentran estudios que focalizan su línea de investigación en indagar cómo con los videojuegos se puede trabajar con el alumnado la resolución colaborativa de problemas. Entre estos trabajos están por ejemplo el etnográfico de Monjelat et al. (2012) con el videojuego Sim City Creator, utilizado en un aula de 30 de educación secundaria de Madrid. Así como el trabajo de Harris (2008) con un juego MMOG titulado Web Earth Online, en el que participan 159 estudiantes de $6^{\circ}$ grado, en California (Estados Unidos).

Ambos estudios obtienen resultados positivos, no obstante, destacan que resulta necesario que el profesorado conozca el juego digital a utilizar y que apoye a alumnos y alumnas durante todo el proceso.

\section{Desarrollo de competencias digitales}

En el artículo de Quesada y Tejedor (2016) se describe una investigación realizada en torno al videojuego World of Warcraft, llevada a cabo para analizar las principales potencialidades educativas de los videojuegos, entre las que se destaca la adquisición de competencias digitales. Bajo dicha finalidad, se llevan a cabo entrevistas con expertas(os), se pasan cuestionarios a exjugadores(as) y jugadores(as), e incluso se graba un documental. En este estudio, "los encuestados destacan haber adquirido a través del juego capacidades como el trabajo en equipo, la mejora de sus reflejos, la agilidad visual, nuevos lenguajes e idiomas y la habilidad de resolver problemas" (Quesada y Tejedor, 2016, p. 194).

\section{Conclusiones}

En relación con la búsqueda bibliométrica realizada a modo de contextualización del estudio, conviene destacar que el tema objeto de estudio en esta investigación está en auge, y muestra de ello es el incremento progresivo de las cifras sobre las publicaciones anuales al respecto. El idioma predominante es el inglés y el país que destaca en productividad 
académica es Estados Unidos, seguido de España y Gran Bretaña. Conviene destacar que a partir de 2013 el número de publicaciones relacionadas aumenta considerablemente.

En cuanto al análisis de contenido realizado, las investigaciones tienden a centrar su ámbito de estudio en el uso, los beneficios y los prejuicios con los que se encuentran las personas con diversidad funcional, trastornos o enfermedades en relación con el uso de videojuegos, y en menor medida en la consideración de estos recursos para responder a un modelo de diseño universal del aprendizaje (DUA).

Se puede afirmar que en la mayoría de las investigaciones se utilizan los videojuegos para la adquisición de competencias, la promoción de la alfabetización digital, el desarrollo emocional o la superación de barreras, pero se dirigen principalmente a colectivos específicos. Esto implica que dichos videojuegos no parten de la perspectiva inclusiva, es decir, aquella en la que conviven y comparten espacios, momentos y experiencias personas con diversidad funcional o trastornos, y sin ellos.

Analizando los resultados, se observa que los videojuegos aparecen vinculados a la atención a la diversidad, tanto para dar respuesta a las necesidades específicas de cierto alumnado, como también a la generación de ciertas circunstancias que pueden influir de forma negativa en el proceso de enseñanza/aprendizaje.

En el caso del alumnado con NEAE (necesidades específicas de apoyo educativo), los videojuegos parece que están siendo usados fundamentalmente para mejorar el bienestar del alumnado, las habilidades sociales, la vida independiente de las personas con TEA, la discapacidad intelectual o las altas capacidades.

En líneas generales, las investigaciones atribuyen efectos muy positivos al desarrollo de distintas competencias en el alumnado con diversidad funcional. No obstante, algunas han introducido importantes cuestionamientos que rompen con la brecha divisoria entre personas con diversidad y las consideradas con desarrollo típico.

En relación con los estudios sobre los posibles efectos perjudiciales ante los altos niveles de uso de videojuegos, los documentos no presentan una clara contundencia al respecto, y en lo que sí parecen coincidir es en la necesidad de limitar el tiempo de uso de estos medios para reducir el riesgo de resultados negativos a largo plazo en cuanto a aspectos como la deserción escolar o la depresión. En este sentido, los trabajos de investigación también parecen evidenciar una relación positiva significativa entre la cantidad de horas que el alumnado pasa jugando a los videojuegos y la falta de atención e hiperactividad en las aulas.

Por otra parte, también encontramos algunos videojuegos que se implementan desde una perspectiva más inclusiva, favoreciendo su acceso y su uso a todas las personas independientemente de su condición. Sin embargo, se echan de menos más investigaciones que analicen el uso de videojuegos en contextos de aprendizaje inclusivo y, sobre todo, que se orienten a favorecer la inclusión educativa. 
Una parte importante de los artículos reflejan resultados que inciden en el papel fundamental de los videojuegos para prevenir o intervenir ante trastornos como la ansiedad, favorecer la motivación, mejorar la calidad de vida o desarrollar capacidades concretas. Por tanto, en respuesta a los objetivos de esta investigación, se puede concluir que existe una gran variedad de videojuegos orientados a colectivos con diversidad de cualquier tipo, destacando especialmente aquellas investigaciones cuyos participantes son personas con TEA, TDAH, discapacidad intelectual, motriz y sensorial. No obstante, todavía es necesario ahondar en la búsqueda de propuestas que aprovechen en mayor medida los videojuegos como oportunidad para la cooperación y el trabajo conjunto entre todas las personas.

Así mismo, en algunas investigaciones se concluye que usar videojuegos en el aula de forma sistemática impulsa en los(as) alumnos(as) el desarrollo de las inteligencias múltiples. Pero también se destaca que, para obtener los objetivos educativos deseados, es necesario que el (la) docente conozca el juego, sepa dinamizar las sesiones y tutorizar al alumnado de forma personalizada, adaptándose a las necesidades que pueda tener cada estudiante. A este respeto, también en la investigación de Garbanzo-Rodríguez et al. (2017) se obtienen mejores resultados si el videojuego se acompaña de instrucción pedagógica.

En cuanto al diseño de los juegos desarrollados para educación, también nos encontramos con que la mayoría son muy parecidos; coincidimos, por tanto, con lo formulado por el Grupo F9 (2000) y por López-Gómez (2017), quienes concluyen que estos juegos incluyen mecánicas demasiado conductistas y superficiales. En general, no atienden a la diversidad de jugadoras y jugadores desde el punto de vista de la accesibilidad, no ofrecen opciones para la modificación o la adaptación. Se trata sobre todo de software lineal y guiado, con escasas opciones para la exploración y no se adaptan a los estilos de aprendizaje del alumnado (Quintero et al., 2009).

Entre las conclusiones de algunas investigaciones, se resalta que queda mucho por explorar en cuanto educación a través de este tipo de videojuegos. En esta misma línea, coincidimos con autores como Stankova et al. (2018), en cuanto a la necesidad de estimular políticas que orienten y apoyen la elaboración de videojuegos para todo el alumnado, especialmente aquel con NEAE. Y es que el fin último de la educación es construir una escuela y una sociedad para todas y todos, y para ello, es fundamental promover la educación inclusiva, también desde la perspectiva digital (Canales y Marqués, 2007).

\section{Referencias}

Ángel, N. (2020). El videojuego y la lectura literaria: nuevo espacio para los nativos digitales. Contextos Educativos: Revista de Educación, 25, 145-159.

Annetta, L. A., Minogue, J., Holmes, S. Y. y Cheng, M. T. (2009). Investigating the impact of video games on high school students' engagement and learning about genetics. Computers \& Education, 53(1), 74-85. 
Area, M., Borrás, J. F. y San Nicolás, M. B. (2015). Educar a la generación de los millennials como ciudadanos cultos del ciberespacio: apuntes para la alfabetización digital. Revista de Estudios de Juventud, 109, 13-32.

Armendarez, J. J. (2015). Video game use among children and adolescents with attention deficit hyperactivity disorder (Publication No. 1604354) [Tesis de maestría, University of Rhode Island]. ProQuest Dissertations and Theses Global. https://www.proquest.com/docview/1739021246/12EBE95F09FC43ECPQ/1?accountid $=17253$

Baldassarri, S., Passerino, L., Ramis, S., Riquelme, I. y Perales, F. J. (2020). Toward emotional interactive videogames for children with autism spectrum disorder. Universal Access in the Information Society, 2020, 1-16. https://doi.org/10.1007/s10209-020-00725-8

Bell, A. y Gresalfi, M. (2017). Teaching with videogames: How experience impacts classroom integration. Technology, Knowledge and Learning, 22(3), 513-526. doi: $10.1007 /$ s10758-017-9306-3

Bourgonjon, J., Valcke, M., Soetaert, R. y Schellens, T. (2010). Students' perceptions about the use of video games in the classroom. Computers \& Education, 54(4), 1145-1156.

Brom, C., Buchtová, M., Šisler, V., Děchtěrenko, F., Palme, R. y Glenk, L. M. (2014). Flow, social interaction anxiety and salivary cortisol responses in serious games: A quasiexperimental study. Computers \& Education, 79, 69-100. https://doi.org/10.1016/j.compedu.2014.07.001

Cabero, J. y Ruiz, J. (2017). Las tecnologías de la información y comunicación para la inclusión: reformulando la brecha digital. Ijeri, International Journal of Educational Research and Innovation, 9, 16-30.

Canales, R. y Marqués, P. (2007). Factores de buenas prácticas educativas con apoyo de las TIC Análisis de su presencia en tres centros educativos. Educar, 39, 115-133.

Cipollone, M. (2015). Motivation to mine: An analysis of the motivation for extended video game play among preadolescents in a physical learning environment (Publication No. 3745843) [Tesis de doctorado, Temple University]. ProQuest Dissertations and Theses Global.

https://www.proquest.com/docview/1861917192/DCF39E40C1574AC8PQ/1?accounti $d=17253$

Contreras, M. I., García-Bauza, C. y Santos, G. (2019). Videogame-based tool for learning in the motor, cognitive and socio-emotional domains for children with intellectual disability. Entertainment Computing, 30. 10.1016/j.entcom.2019.100301

Corrales-Astorgano, M., Martínez-Castilla, P., Escudero-Mancebo, D., Aguilar, L., González- 
Ferreras, C. y Cardeñoso-Payo, V. (2019). Automatic assessment of prosodic quality in down syndrome: Analysis of the impact of speaker heterogeneity. Applied Sciences, 9(7). http://dx.doi.org/10.3390/app9071440

Cortés, S., García, M. R. y Lacasa, P. (2012). Videojuegos y redes sociales. El proceso de identidad en Los Sims 3. RED, Revista de Educación a Distancia, 33. https://revistas.um.es/red/article/view/233141

Cusmano, G. (2010). An investigation of the relationship between violent video games and self-reported aggression (Publication No. 3467101) [Tesis de doctorado, Nova Southeastern University]. ProQuest Dissertations and Theses Global. https://www.proquest.com/docview/1018476599/B8D7E45548A94430PQ/1?accounti $d=17253$

Cho, I. Y. K., Jelinkova, K., Schuetze, M., Vinette, S. A., Rahman, S., McCrimmon, A., Deborah, D. y Bray, S. (2017). Circumscribed interests in adolescents with autism spectrum disorder: A look beyond trains, planes, and clocks. PLoS One, 12(11). http://dx.doi.org/10.1371/journal.pone.018741

Demirtas, S., Ulasb, O. y Kizildagc, S. (2015). Relation between video game addiction and interfamily. Relationships on primary school students. Educational Sciences: Theory \& Practice, 15(2), 489-497. http://dx.doi.org/10.12738/ESTP.2015.2.2090

Dos Santos-Venturelli, A. y Denise-Pereira, A. F. (2019). A visão do professor sobre jogos digitais no ensino da matemática para alunos com deficiência intelectual: Estado da arte - Teacher's view about digital games in mathematics education for students with intellectual disabilities: State of art. Educação Matemática Pesquisa, 21(1). http://dx.doi.org/10.23925/1983-3156.2019v21i1p180-19

Earp, J., Ott, M., Popescu, M., Romero, M. y Usart, M. (2014). Supporting human capital development with serious games: An analysis of three experiences. Computers in Human Behavior, 30, 715-720.

Franceschini, S., Trevisan, P., Ronconi, L., Bertoni, S., Colmar, S., Double, K., Facoetti, A. y Gori, S. (2017). Action video games improve reading abilities and visual-to-auditory attentional shifting in english-speaking children with dyslexia. Scientific Reports (Nature Publisher Group), 7, 1-12. http://dx.doi.org/10.1038/s41598-017-05826-8

Gálvez, A. (2002). Revisión bibliográfica: usos y utilidades. Matronas Profesión, 3(10), 25-31. Garbanzo-Rodríguez, G., Rivera-Villareal, C., Smith-Castro, V. y Pérez-Sánchez, R. (2017). Efectos de un videojuego de simulación sobre tendencias altruistas en un contexto de educación abierta. Revista Colombiana de Educación, 73, 41-57. https://doi.org/10.17227/01203916.73rce39.56 
García-Redondo, P., García, T., Areces, D., Núñez, J. C. y Rodríguez, C. (2019). Serious games and their effect improving attention in students with learning disabilities. International Journal of Environmental Research and Public Health, 16(14), 2480. http://dx.doi.org/10.3390/ijerph16142480

Gerling, K. M., Linehan, C., Kirman, B., Kalyn, M. R., Evansa, A. B. y Hicks, K. C. (2015). Creating wheelchair-controlled video games: Challenges and opportunities when involving young people with mobility impairments and game design experts. International Journal of Human-Computer Studies, 94, 64-73. https://doi.org/10.1016/j.ijhcs.2015.08.009

González, C. V. (2020). Herramientas TIC para la gamificación en Educación Física. Edutec, Revista Electrónica de Tecnología Educativa, 71, 67-83.

Gros, B. (2007). Digital games in education: The design of games-based learning environments. Journal of Research on Technology in Education, 40(1), 23-38.

Grupo F9 (2000). Jugar con el ordenador, también en la escuela. Cuadernos de Pedagogía, 291, 52-54.

Guerra, J. y Revuelta, F.I. (2015). Videojuegos precursores de emociones positivas: propuesta metodológica con Minecraft en el aula hospitalaria. IJERI, International Journal of Educational Research and Innovation, 3, 105-120. https://www.upo.es/revistas/index.php/IJERI/article/view/1447

Guerrero, B. y Valero, L. (2013). Efectos secundarios tras el uso de realidad virtual inmersiva en un videojuego. International Journal of Psychology and Psychological Therapy, 13(2), 163-178. https://www.ijpsy.com/volumen13/num2/353/efectos-secundariostras-el-uso-de-realidad-ES.pdf

Harris, D. (2008). A comparative study of the effect of collaborative problem-solving in a Massively Multiplayer Online Game (MMOG) on individual achievement (Publication No. 3317694) [Tesis doctoral, University of San Francisco]. ProQuest Dissertations and Theses

Global. https://www.proquest.com/docview/304825929/fulltextPDF/30020F466301424BPQ/1 ?accountid $=17253$

Hillier, K. (2013). Effects of Wii Game console on increased engagement and peer interactions (Publication No. 1542389) [Tesis de máster, University of Washington]. ProQuest Dissertations and Theses Global. https://www.proquest.com/docview/1426400353/16B82633F2874068PQ/1 ?accountid $=17253$

Holguin, J., Taxa, F., Flores, R. y Olaya, S. (2020). Proyectos educativos de gamificación por 
videojuegos: desarrollo del pensamiento numérico y razonamiento escolar en contextos vulnerables. EDMETIC, Revista de Educación Mediática y TIC, 9(1), 80-103. https://doi.org/10.21071/edmetic.v9i1.12222

Jasem, A. Z. y Delport, S. M. (2019). Mothers' perspectives on the play of their children with attention deficit hyperactivity disorder. Occupational Therapy International, 2019, ID 6950605. https://doi.org/10.1155/2019/6950605

Jaume, A., Varona, J., Moyà, G. y Perales, F.J. (2013). Rehabilitación motivacional basada en la utilización de Juegos Serios. Virtual Archaeology Review, 4(9), 167-173. https://doi.org/10.4995/var.2013.4270

Kaigo, M. y Okura, S. (2020). Game accessibility and advocacy for participation of the $\begin{array}{llll}\text { japanese disability } & 162 .\end{array}$ http://dx.doi.org/10.3390/info11030162

Kasper, D., Welsh, S. y Chambliss, C. (1999). Educating students about the risks of excessive videogame usage. http://files.eric.ed.gov/fulltext/ED426315.pdf

Kneifer, C. J. (2014). A comparison study on violent video games: Explained by the gamers themselves (Publication No. 1563329) [Tesis de máster, University of South Florida]. ProQuest Dissertations and Global. https://www.proquest.com/docview/1596023232/C27B0F40342142A6PQ/1?accounti $\mathrm{d}=17253$

Lázaro, J., Estebanell, M. y Tedesco, J. (2015). Inclusión y cohesión social en una sociedad digital. RUSC, Universities and Knowledge Society Journal, 12(2), 44-59. $10.7238 /$ rusc.v12i2. 2459

Ley Orgánica 8/2013 de mejora de la calidad educativa (LOMCE). Boletín Oficial del Estado, 295, 97858-97921.

Ley Orgánica 3/2020, de 29 de diciembre, por la que se modifica la Ley Orgánica 2/2006, de 3 de mayo, de educación (LOMLOE). Boletín Oficial del Estado, 340, 122868-122953.

Liu, C. (2014). Long term effects of video and computer game heavy use on health, mental health and education outcomes among adolescents in the U.S. (Publication No. 3673789) [Tesis de doctorado, University of Illinois at Urbana-Champaign]. ProQuest Dissertations and Theses Global. https://www.proquest.com/docview/1652503789/fulltextPDF/480695ACDFBC4224PQ $/ 1$ ?accountid=17253

López-Gómez, S. (2017). Análise descritiva e interpretativa do deseño e contido dos videoxogos elaborados en Galicia [Tesis de doctorado, Universidade de Santiago de Compostela]. Repositorio Universidade de Santiago de Compostela. 
https://minerva.usc.es/xmlui/handle/10347/16695

López-Rodríguez, M.M., Fernández-Millan, A., Ruiz-Fernández, M. D., Dobarrio-Sanz, I. y Fernández-Medina, I. M. (2020). New technologies to improve pain, anxiety and depression in children and adolescents with cancer: A systematic review. International Journal of Environmental Research and Public Health, 17(10), 3563. http://dx.doi.org/10.3390/ijerph17103563

Lozano, J., Castillo, I. S. y Veas, A. (2014). Alumnado con necesidades específicas de apoyo educativo de educación secundaria obligatoria y el consumo de las TIC en la región de Murcia. Étic@net, 1(14), 57-69. http://hdl.handle.net/10045/46094

Lyon, N. E. (2015). Feeling factory: A prosody improvement game for children with ASD (Publication No. 1594280) [Tesis de máster, Drexel University]. ProQuest Dissertations and Theses Global. https://www.proquest.com/docview/1706908703/BB98AA4052F44659PQ/1?accounti $d=17253$

Maras, D., Flament, M. F., Murray, M., Buchholz, A., Henderson, K. A., Obeid, N. y Goldfield, G. S. (2015). Screen time is associated with depression and anxiety in Canadian youth. Preventive Medicine, 73, 133-138. https://doi.org/10.1016/j.ypmed.2015.01.029

Marín, V. y Ramírez, A. (2012). Posibilidades educativas de los videojuegos y juegos digitales en educación inclusiva. En V. Marín (coord.), Los videojuegos y los juegos digitales como materiales educativos (pp. 165-191). Síntesis.

Mathieu, S., Barrault, S., Brunault, P. y Varescon, I. (2020). The role of gambling type on gambling motives, cognitive distortions, and gambling severity in gamblers recruited online. PLoS One, 15(10). http://dx.doi.org/10.1371/journal.pone.0238978

Mejía, J. A., Hernández, G., Toledo, C., Mercado, J., Vera, A., Leija, L. y Gutiérrez, J. (2019). Upper limb rehabilitation therapies based in videogames technology review. En 2019 Global Medical Engineering Physics Exchanges/Pan American Health Care Exchanges (GMEPE/PAHCE) (pp. 1-5). Institute of Electrical and Electronics Engineers.

Monjelat, N., Méndez, L. y Lacasa, P. (2012). Procesos de resolución de problemas y videojuegos: el caso de Sim City Creator. Revista Electrónica de Investigación $\begin{array}{llll}\text { Psicoeducativa } \quad y & \text { Psicopedagógica, } & 10(28), & 1493-1522 .\end{array}$ http://dx.doi.org/10.25115/ejrep.v10i28.1543

Navaneetham, J. y Chandran, J. (2018). Video game use among schoolchildren and its impact on the study habits. Indian Journal of Social Psychiatry, 34(3), 208-212. http://dx.doi.org/10.4103/ijsp.ijsp_58_17

Pagès, J. (2009). Competencia social y ciudadana. Aula de Innovación Educativa, 187, 7-11. 
Papa Gusho, L. y Mitrushi, M. (2017). The manifestation of disturbing behavior on adolescents as a result of using violent video-games. Academicus, 16, 113-121. http://dx.doi.org/10.7336/academicus.2017.16.08

Puig, M. y Morales, J. A. (2015). La formación de ciudadanos: conceptualización y desarrollo de la competencia social y cívica. Educación XXI, 18(1), 259-282. https://doi.org/10.5944/educxx1.18.1.12332

Quesada, A. y Tejedor, S. (2016). Aplicaciones educativas de los videojuegos: el caso de World of Warcraft. Pixel-Bit (Revista de Medios y Educación), 48, 187-196. https://doi.org/10.12795/pixelbit.2016.i48.12

Quintero, J., Munévar, F. I. y Álvarez, D. Y. (2009). Ambientes naturales y ambientes virtuales de aprendizaje. Revista Colombiana de Educación, 56, 12-37. https://doi.org/10.17227/01203916.7579

Ramos-Ramírez, R. y Mauricio, D. (2019). Videogame to support the teaching of reading to deaf children using gamification. Revista Ibérica de Sistemas e Tecnologias de Informação, 23, 145-157. https://search.proquest.com/docview/2348877991 ?accountid=17197

Ren, Z. y Wu, J. (2019). The effect of virtual reality games on the gross motor skills of children with cerebral palsy: A meta-analysis of randomized controlled trials. International Journal of Environmental Research and Public Health, 16(20). http://dx.doi.org/10.3390/ijerph16203885

Reyes, R. y Prado, A. B. (2020). Las tecnologías de información y comunicación como herramienta para una educación primaria inclusiva. Revista Educación, 44(2) 479-497.

Reyes-Hernández K. L., Sánchez-Chávez N. P., Toledo-Ramírez M. I., Reyes-Gómez, U., Reyes-Hernández, D. P. y Reyes-Hernández, U. (2014). Los videojuegos: ventajas y perjuicios para los niños. Revista Mexicana de Pediatría. 81(2), 74-78.

Reyes-Padilla, R. M., Padrón-Morales, F. D., Soto-Luévano, J. E. y Arévalo-Mercado, C. A. (2020). Videojuegos y su impacto en la salud mental: estudio experimental sobre exposición a videojuegos violentos en estudiantes universitarios. Tecnología Educativa Revista Conaic, 7(1), 24-30.

Rodríguez, M. y Arroyo, M. (2014). Las TIC al servicio de la inclusión educativa. Digital Education Review, 25, 108-126.

Rodríguez-Jiménez, M. (2015). Desarrollo de las funciones ejecutivas a través de videojuegos en la atención a la diversidad [Tesis doctoral, Universidad de Extremadura]. Repositorio Institucional Universidad de Extremadura. http://dehesa.unex.es/handle/10662/4162 Romera, E. M., Ortega, R. y Monks, C. (2008). Impacto de la actividad lúdica en el desarrollo 
de la competencia social. Internacional Journal of Psychology and Psychological Therapy, 8(2), 193-202.

Saldarriaga, P. J., Bravo, G. D. R. y Loor, M. R. (2016). La teoría constructivista de Jean Piaget y su significación para la pedagogía contemporánea. Dominio de las Ciencias, 2(3), 127-137.

Sampedro, B. (2012). La perspectiva psicológica del videojuego en la infancia y la adolescencia. En V. Marín (coord.), Los videojuegos y los juegos digitales como materiales educativos (pp. 35-60). Síntesis.

Sampedro, B. y McMullin, K. J. (2015). Videojuegos para la inclusión educativa. Digital Education Review, 27, 122-137.

Santos, M. A., Cernadas, F. X. y Lorenzo, M. M. (2014). La inclusión educativa de la inmigración y la formación intercultural del profesorado. Revista Electrónica Interuniversitaria de Formación del Profesorado, 17(2), 123-137. http://dx.doi.org/10.6018/reifop.17.2.196931

Sefen, J. A. N., Al-Salmi, S., Shaikh, Z., AlMulhem, J. T., Rajab, E. y Fredericks, S. (2020). Beneficial use and potential effectiveness of physical activity in managing autism spectrum disorder. Frontiers in Behavioral Neuroscience. http://dx.doi.org/10.3389/fnbeh.2020.587560

Shaffer, D. W. (2006). Epistemic frames for epistemic games. Computers \& Education, 46(3), 223-234.

Sproull, C. (2011). The impact of a digital role playing game on the executive functioning skills of students with ADHD (Publication No. 3487722) [Tesis de doctorado, Walden University]. ProQuest Dissertations and Theses Global. https://www.proquest.com/docview/913389042/fulltextPDF/E1D3A492F073487DPQ/ 1 ? accountid $=17253$

Stankova, M., Ivanova, V. y Kamenski, T. (2018). Use of educational computer games in the initial assessment and therapy of children with special educational needs in Bulgaria. TEM Journal, 7(3), 488.

Tobar, H., Fabregat, R. y Baldiris, S. (2015). Augmented reality game-based learning for mathematics skills training in inclusive contexts. IE Comunicaciones (Revista $\begin{array}{lll}\text { Iberoamericana de Informática } & 21 .\end{array}$ https://dialnet.unirioja.es/servlet/articulo?codigo $=5113267$

Tomás-Górriz, V. y Tomás-Casterá, V. (2018). La bibliometría en la evaluación de la actividad científica. Hospital a Domicilio, 2(4), 145-163.

Uz, C. y Cagiltay, K. (2015). Social interactions and games. Digital Education Review, 27, 1- 
12. https://files.eric.ed.gov/fulltext/EJ1064986.pdf

Vidal, M. I., López, M., Marín. D. y Peirats, J. (2017). Revisión y análisis de investigación publicada sobre intervención gamificada en discapacidad intelectual. Etic@net, Revista Científica Electrónica de Educación y Comunicación en la Sociedad del Conocimiento, 18(2), 274-297.

Wallace, J. (2012). Video game habits and their impact on attentional functioning (Publication No. 3507092) [Tesis de doctorado, Fairleigh Dickinson University]. ProQuest Dissertations and Theses Global. https://www.proquest.com/docview/1015162979/fulltextPDF/879AC67E810A4125PQ/ 1 ? accountid $=17253$

Wartberg, L., Zieglmeier, M. y Kammerl, R. (2020). An empirical exploration of longitudinal predictors for problematic internet use and problematic gaming behavior. Psychological Reports, 124(2), 543-554.

Zhao, Z. y Linaza, J. L. (2015). La importancia de los videojuegos en el aprendizaje y el desarrollo de niños de temprana edad. Electronic Journal of Research in Educational Psychology, 13(36), 301-318. http://dx.doi.org/10.14204/ejrep.36.14018 